\title{
Metastatic Melanoma With Sebocyte-Like Melanocytes and Widespread Visceral Involvement
}

\author{
Li-Wei Chang, ${ }^{1}$ Viktoryia Kazlouskaya, ${ }^{1}$ Jenna R. Bordelon, ${ }^{1}$ Marion A. Hughes, ${ }^{2}$ Yana G. Najjar, ${ }^{3}$ \\ Yuri Bunimovich, ${ }^{1}$ Arivarasan Karunamurthy, ${ }^{1}$ Jonhan $\mathrm{Ho}^{1}$
}

\begin{abstract}
1 Department of Dermatology, University of Pittsburgh Medical Center, Pittsburgh, PA, USA
2 Department of Radiology, University of Pittsburgh Medical Center, Pittsburgh, PA, USA

3 Department of Medicine, University of Pittsburgh Medical Center, Pittsburgh, PA, USA
\end{abstract}

Key words: sebocyte-like melanocyte, metastatic melanoma, clear cell melanocyte, melanocyte, malignant melanoma

Citation: Chang LW, Kazlouskaya V, Bordelon JR, Hughes MA, Najjar YG, Bunimovich Y, Karunamurthy A, Ho J. Metastatic melanoma with sebocyte-like melanocytes and widespread visceral involvement. Dermatol Pract Concept. 2020;10(2):e2020045. DOI: https://doi. org/10.5826/dpc.1002a45

Accepted: January 30, 2020; Published: April 20, 2020

Copyright: $\odot 2020$ Chang et al. This is an open-access article distributed under the terms of the Creative Commons Attribution License, which permits unrestricted use, distribution, and reproduction in any medium, provided the original author and source are credited.

Funding: None.

Competing interests: The authors have no conflicts of interest to disclose.

Authorship: All authors have contributed significantly to this publication.

Corresponding author: Li-Wei Chang, MD, DSc, Department of Dermatology, University of Pittsburgh Medical Center, 3708 Fifth Avenue, 5th Floor, Suite 500.68, Pittsburgh, PA 15213, USA. Email: chang1@upmc.edu

\section{Introduction}

Melanocytes may assume a variety of shapes: epithelioid, round, spindled, pagetoid, dendritic, and with clear cytoplasm, among others. A type of melanocyte with multivacuolated cytoplasm surrounding a dark-stained scalloped nucleus was initially described by Eftychiadis et al as "sebocyte-like" [1]. These melanocytes are more commonly seen in benign congenital nevi, but also have been rarely reported in malignant melanoma, with only a few case reports in the literature [2]. The clear cell change is believed to result from intracellular enlargement of melanosomes with separation of their fibers. Such neoplasms may mimic sebaceous neoplasms and clear cell carcinoma. We report a case of a metastatic melanoma with undifferentiated cells exhibiting sebaceous-like features.

\section{Case Presentation}

A 44-year-old woman with no significant medical history and no family history of melanoma presented with numerous firm subcutaneous nodules on her scalp, trunk, and extremities. The patient first noticed a nodule on her left upper arm 6 months prior, and then similar nodules arose diffusely. The patient endorsed fatigue, dyspnea, dysphagia, epigastric pain, right-sided weakness, and right leg pain. Physical examination revealed numerous 1- to 6-cm, firm, flesh-colored to red subcutaneous nodules diffusely on the scalp, neck, trunk, and extremities (Figure 1). A total body skin examination did not identify lesions of concern for primary malignancy. Brain MRI and a whole-body CT scan revealed widespread soft tissue metastases, with innumerable lesions in the brain, chest wall, liver, spleen, and bone (Figure 2). 
Skin biopsies taken from 2 nodules both revealed a deep dermal tumor composed of sheets of densely arranged atypical cells (Figure 3). Morphologically, many cells had scalloped nuclei and "bubbly" cytoplasm resembling sebaceous cells (Figure 4). The cells expressed SOX10 (Figure 5) and were negative for pancytokeratin. EWSR1 fusion gene study, to rule out clear cell sarcoma, was negative. A diagnosis of metastatic melanoma was made; $B R A F^{V 600 E}$ mutation was identified. In addition, the patient had a pericardial effusion requiring a pericardial window and melanoma cells were found in the pericardial fluid. The patient was then started on encorafenib and binimetinib, and within 72 hours her cutaneous lesions were visibly regressing. A subsequent whole-body imaging after 2 months of treatment demonstrated marked reduction in the size and number of subcutaneous nodules in the thoracic and abdominal wall, as well as all visceral lesions. The majority of brain parenchymal lesions also decreased in size. At 4-month follow-up, the patient continued to do well clinically, but brain MRI demonstrated progression of metastatic disease.

\section{Conclusions}

The implication of sebocyte-like features on the prognosis of melanoma is not well established. Few other cases have been reported, and none were as rapidly progressive as in this patient [2]. BRAF V600E mutation occurs in 50\% of melanomas and has historically been associated with more aggressive disease. Treatment with combined BRAF and MEK inhibitors carries a high response rate and is therefore often first-line in those with high disease burden. We present a case of melanoma with sebocyte-like features that carries $B R A F^{V 600 E}$ mutation and significantly responded to combination targeted therapy.

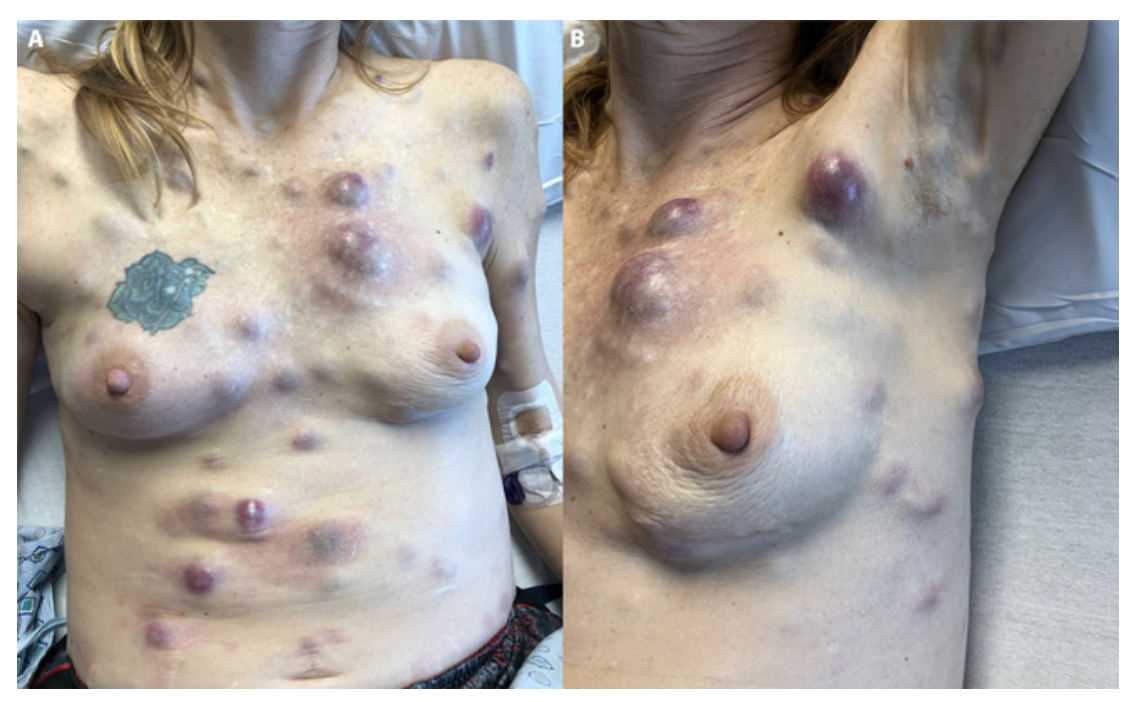

Figure 1. Multiple tender, firm, subcutaneous nodules on the trunk (A) with involvement of left axilla (B).
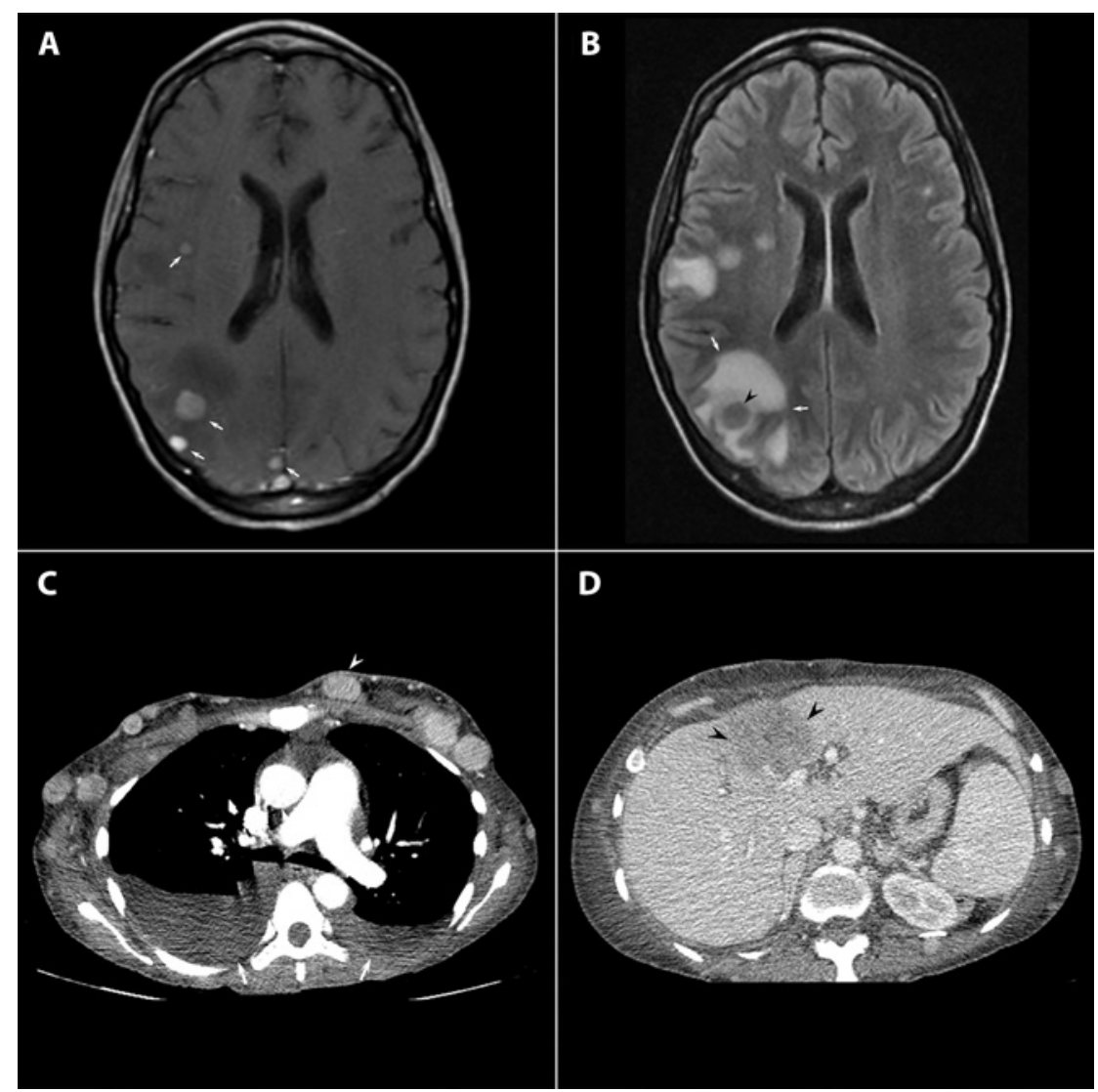

Figure 2. Radiology findings. (A) Axial T1 weighted image with contrast demonstrates multiple enhancing intracranial lesions (white arrows) within the right frontal and parietal lobe consistent with metastases. (B) Axial fluid-attenuated inversion recovery (FLAIR) images demonstrate varying degrees of vasogenic edema (white arrows) surrounding the brain metastases, most notably the right parietal lesion (black arrowhead). (C) Axial CT image of the chest demonstrates multiple subcutaneous metastatic nodules (white arrowhead on a representative nodule). Bilateral pleural effusions (white arrows) are also noted. (D) Axial CT image of the abdomen with contrast demonstrates the large hypoenhancing left hepatic lobe lesion, compatible with a hepatic metastasis (black arrowheads). 


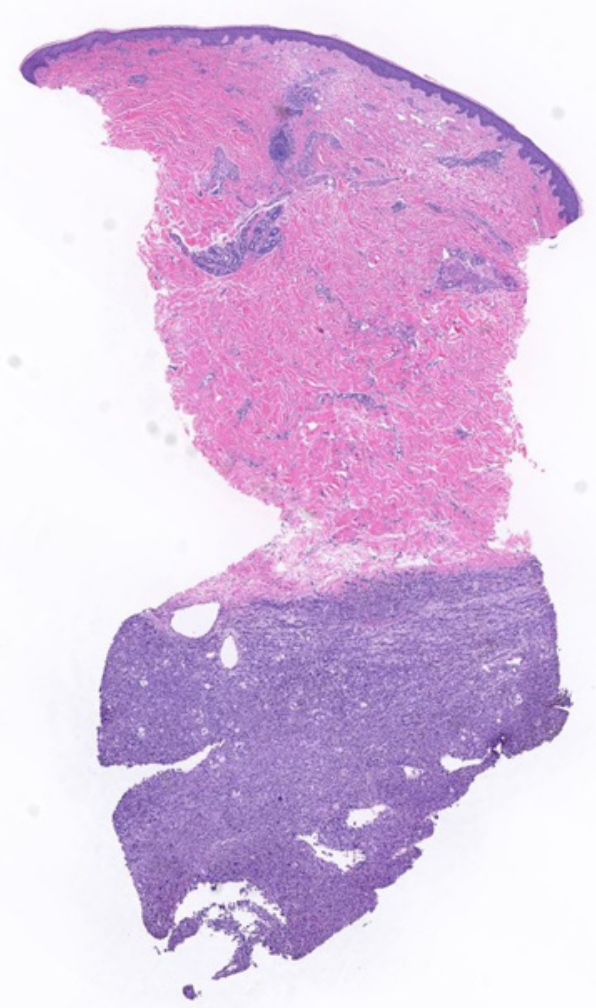

Figure 3. Dermal proliferation with sheets of undifferentiated cells. H\&E staining. Scanned slide, cropped image.

\section{References}

1. Eftychiadis AS, Iozzo RV, Ackerman AB. Sebocyte-like melanocytes in melanocytic nevi. Dermatopathol Pract Concept. 1996;2:49-50.

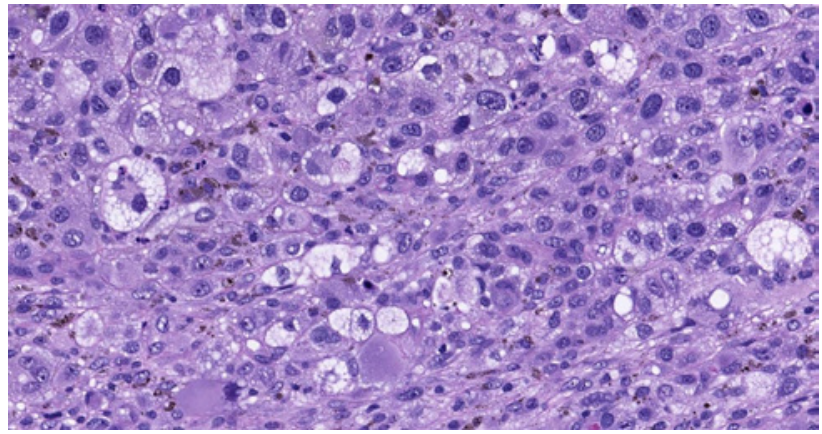

Figure 4. Higher power shows undifferentiated cells with sebocyte-like morphology and melanin pigment. H\&E staining. Scanned slide, cropped image.

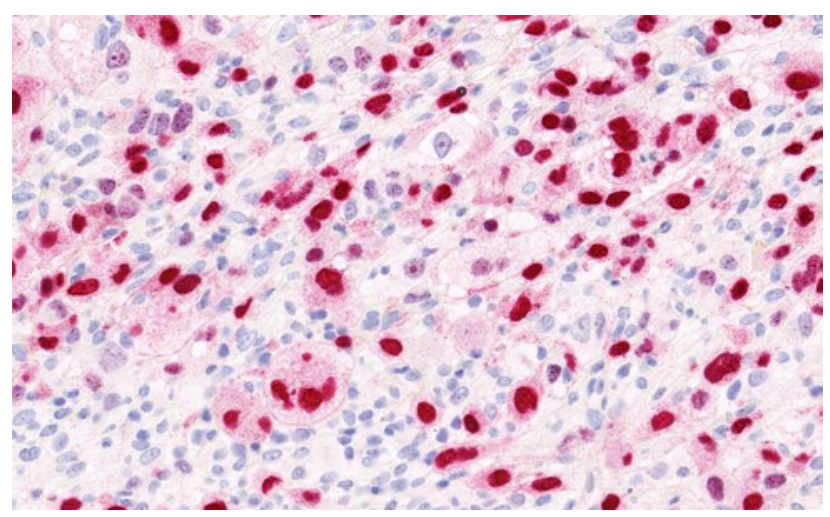

Figure 5. Sebocyte-like cells in the dermal tumor express SOX10. SOX10 immunostaining, cropped image.

2. Molina-Ruiz AM, Ortiz-Reina S, Carranza C, Kutzner H, Requena L. Sebocyte-like cell primary cutaneous melanoma: a rare cytologic variant of malignant melanoma. Am J Dermatopathol. 2015;37(11):862-865. 